\title{
Erratum to: Interaction of the signaling state analog and the apoprotein form of the orange carotenoid protein with the fluorescence recovery protein
}

\author{
Marcus Moldenhauer $^{1} \cdot$ Nikolai N. Sluchanko $^{2,6} \cdot$ Neslihan N. Tavraz $^{1} \cdot$ Cornelia Junghans $^{1} \cdot$ David Buhrke $^{1}$ • \\ Mario Willoweit ${ }^{1} \cdot$ Leonardo Chiappisi $^{3} \cdot$ Franz-Josef Schmitt $^{1} \cdot$ Vladana Vukojević $^{4} \cdot$ Evgeny A. Shirshin $^{5}$. \\ Vladimir Y. Ponomarev $^{6} \cdot$ Vladimir Z. Paschenko $^{6} \cdot$ Michael Gradzielski $^{3} \cdot$ Eugene G. Maksimov $^{6}$. \\ Thomas Friedrich ${ }^{1}$
}

Published online: 19 September 2017

(c) Springer Science+Business Media B.V. 2017

\section{Erratum to: Photosynth Res}

DOI 10.1007/s11120-017-0346-2

In Fig. 1a in the original article, the amino acid side chains were incorrectly labeled in the structure representation of the orange carotenoid protein (OCP). The corrected Fig. 1 is printed in this erratum.

The online version of the original article can be found under doi:10.1007/s11120-017-0346-2.

Thomas Friedrich

friedrich@chem.tu-berlin.de

1 Institut für Chemie Sekr. PC 14, Technische Universität Berlin, Straße des 17. Juni 135, 10623 Berlin, Germany

2 A.N. Bach Institute of Biochemistry, Federal Research Center "Fundamentals of Biotechnology", Russian Academy of Sciences, 33 Leninsky prospect, building 1, Moscow, Russian Federation 119071

3 Institut für Chemie Sekr. TC 7, Technische Universität Berlin, Straße des 17. Juni 124, 10623 Berlin, Germany

4 Department of Clinical Neuroscience, Center for Molecular Medicine, Karolinska Institutet, CMM L8:01,

17176 Stockholm, Sweden

5 Department of Quantum Electronics, Faculty of Physics, M.V. Lomonosov Moscow State University, Leninskie Gory, Moscow, Russian Federation 119992

6 Department of Biophysics, Faculty of Biology,

M.V. Lomonosov Moscow State University, Leninskie Gory,

1, p. 12, Moscow, Russian Federation 119992 


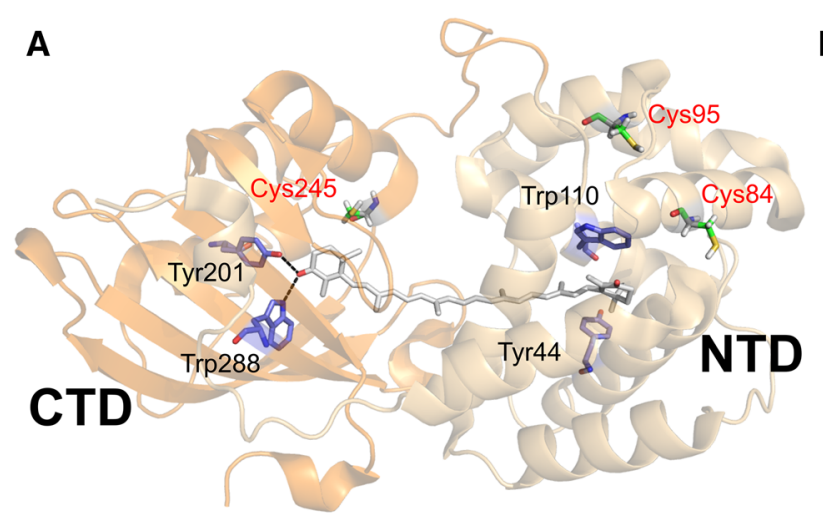

B
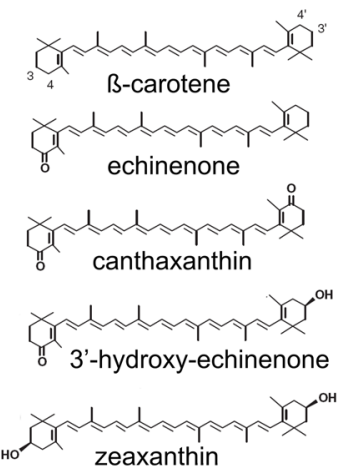

C

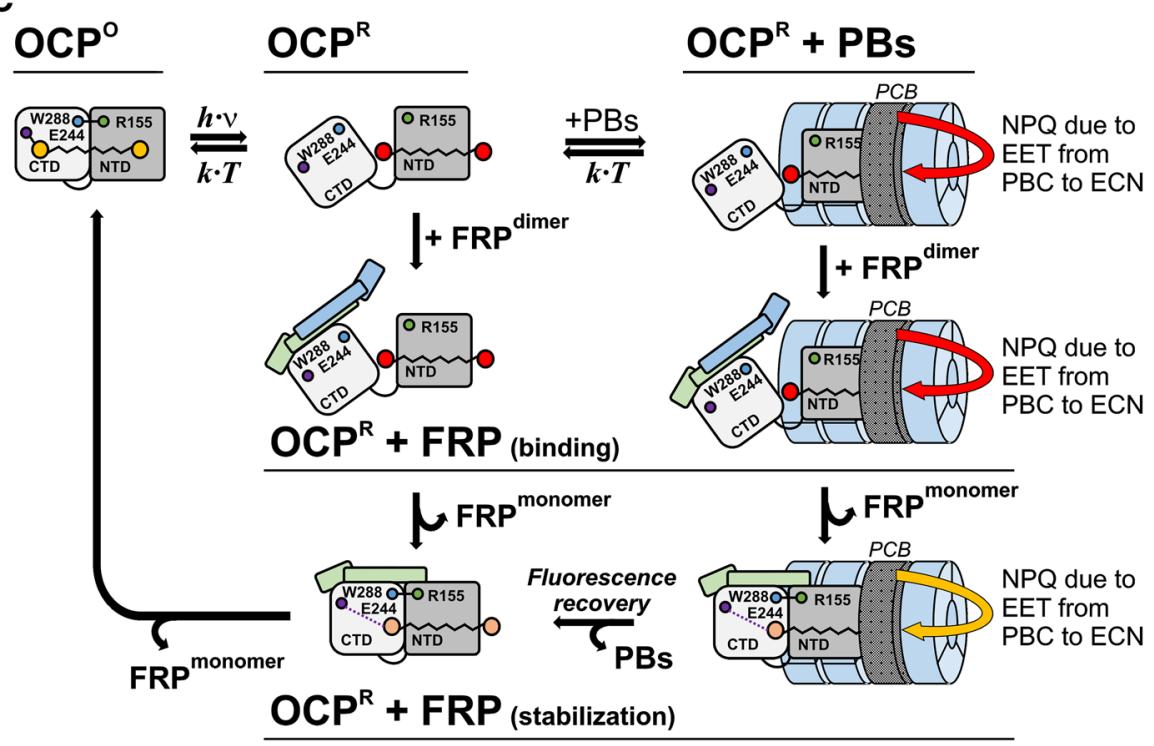

Fig. 1 Structure of the OCP protein and scheme for the interplay of OCP with PBs and FRP during NPQ. a OCP crystal structure (PDB entry 4XB5, (Leverenz et al. 2015)) with the canthaxanthin cofactor shown in stick representation. OCP is divided into an N-terminal and a C-terminal domain (NTD, CTD), with Trp288 and Tyr201 in the CTD involved in $\mathrm{H}$-bond interactions (black dashed lines) to the 4-keto group of one of the $\beta$-rings. Tyr44 and Trp110 in the NTD are also involved in carotenoid coordination. The three cysteines in Synechocystis OCP are also shown (Cys84 and Cys95 in the NTD and Cys245 in the CTD). b Chemical structures of some carotenoids mentioned in this work. c Scheme of the major stages of NPQ. Upon absorption of blue-green light, the orange form of OCP $\left(\mathrm{OCP}^{\mathrm{O}}\right)$ is photoconverted into the active red state $\left(\mathrm{OCP}^{\mathrm{R}}\right)$ (Wilson et al. 2008). Consequently, NTD and CTD dissociate, the salt bridge between Glu244 (CTD) and Arg155 (NTD) breaks and the carotenoid trans- locates into the NTD. NPQ activation in vivo is limited by the rate at which the $\mathrm{OCP}^{\mathrm{R}}$ binds to PBs (Gorbunov et al. 2011; Maksimov et al. 2015a). During this dark phase of NPQ activation, $\mathrm{OCP}^{\mathrm{R}}$ forms a stable complex with PBs, leading to PBs fluorescence quenching (Maksimov et al. 2014). Alternatively, $\mathrm{OCP}^{\mathrm{R}}$ can spontaneously reconvert into the $\mathrm{OCP}^{\mathrm{O}}$ form, or form a complex with FRP. The presence of FRP leads to an almost 10 -fold increase of the $\mathrm{OCP}^{\mathrm{R}}-\mathrm{OCP}^{\mathrm{O}}$ conversion rate (Boulay et al. 2010). Finally, under low-light conditions, OCP uncouples from PBs and energy flow from PBs to the photosynthetic reaction centers is restored. Of note, FRP exists in a dimeric form that becomes monomeric upon interaction with the $\mathrm{OCP}^{\mathrm{R}}$ form (Sluchanko et al. 2017). Therefore, the interaction between OCP and FRP involves FRP dimer dissociation prior to or during binding to $\mathrm{OCP}^{\mathrm{R}}$ 